\title{
REVIEW OF WIRELESS MIMO CHANNEL MODELS
}

\author{
C. L. Anioke ${ }^{1}$, C. O. Nnamani ${ }^{2}$ and C. I. Ani $^{3}$ \\ 1,2,3 Department of Electronic ENGineERING, University of Nigeria NSUKKa, ENUGU STATE. NiGERIA \\ E-mail addresses: 1chidera.anioke@unn.edu.ng, 2obinna.nnamani@unn.edu.ng, 3 cosmas.ani@unn.edu.ng
}

\begin{abstract}
The need to increase spectral efficiency has led to the design of multiple antenna systems for both transmit and receive sides otherwise known as MIMO. Channel modeling forms an integral part of this design. Therefore it is very important to investigate and understand existing MIMO channel models. This paper provides a detailed review of existing MIMO channel models, their characteristics, tradeoffs and challenges. As with most models in the scientific and technical fields, open issues in MIMO channel modeling have also been enumerated.
\end{abstract}

Keywords: MIMO, channel, modelling, scatters, transmit, receive

\section{INRODUCTION}

Advancement in wireless technologies and antenna array configurations has increased in the volume of ongoing researches in wireless channel modeling. The communication channel represents a major component that determines system performance. Modeling of channels is essential for the design of wireless systems. The need to improve capacity, increase spectral efficiency and minimize the effects of multipath interference has resulted in multiple antenna systems at both ends of a communication link otherwise known as multiple-input-multiple-output (MIMO) systems. The MIMO system operates at a level of complexity that exploits channel space-time resources required to access the potential performance of practical multi-antenna links [1].In a MIMO wireless system, the transmitted signal interacts with the environment in a very complex way. This is as a result of reflections, diffractions and signal scattering resulting from objects/scatterers in the signal path and electromagnetic waves around objects. A continuous succession of paths is produced by each scatterer joining the transmitter and the receiver. Thus, a channel model for non-line of sight (NLOS) propagation must involve details about the multipath environment. In a multipath environment, the communication link can be described by properties such as amplitude fade variations, carrier phase; time delay spread information, power delay profile, angle of arrival and departure, Doppler shift and the number of multipath components [2]. Consequently, realistic assumptions could be made so that postulated channel models could facilitate performance assessment of potential space-time coding approaches for practical propagation scenarios. This paper provides a detailed review of the MIMO multipath channel models. The review includes the characterization of the MIMO channel, model classifications and their applications to different environments as well as MIMO mobility models. Finally open issues on MIMO channel modeling were discussed.

\subsection{Characterization of the Mimo Channel}

With the advent of the MIMO wireless systems, there arose a need for spatial channel models that will suit a communication link having both transmit and receive diversity. The modeling of the spatial channel impulse response is vital for the simulation of the MIMO communication channel. The MIMO channel may be characterized in terms of the propagation environment or in terms of the MIMO system circuitry (antenna configuration, antenna type, antenna pattern, filter mechanisms e.t.c.). Characterizing the MIMO channel impulse response in terms of the former case is known as double-directional channel impulse response whereas the latter is referred to as the MIMO channel matrix $[1,2,3]$. In order to determine the spatial or directional information of the MIMO channel, channel models must include parameters for the Angle of Arrival (AoA), the Angle of 
Departure (AoD) and polarization of the multipath components [4, 8]. The MIMO channel impulse response is a summation of several multipath components each of which has its own amplitude, phase, delay, AOA and AOD. The distribution of these parameters is dependent on the type of propagation environment being modeled. The properties of the propagation environment can be defined by representing $h$ as a function of some of key channel parameters. Assume $h$ to be a time varying impulse response with delay $\tau$. Therefore $h$ can be expressed as $h\left(t, \tau, \theta_{R}, \varphi_{R}, \theta_{T}, \varphi_{T}\right)$ with an elevation angle taken with respect to the coordinate frame of transmit and receive array $\left(\theta_{T}, \theta_{R}\right)$, azimuthal angle referenced to transmit and receive array coordinate frame $\left(\varphi_{T}, \varphi_{R}\right)$. Assume that all scattering in the propagation channel are in the far-field and that a discrete number of propagation paths, $L$, connect transmit and receive arrays. The channel impulse response can be expressed as in (1)

$$
\begin{aligned}
h\left(t, \tau, \theta_{R}, \varphi_{R}, \theta_{T},\right. & \left.\varphi_{T}\right) \\
& =\sum_{l=1}^{L} A_{l} \delta\left(\tau-\tau_{l}\right) \delta\left(\theta_{T}-\theta_{T, l}\right) \\
& \times \delta\left(\varphi_{T}-\varphi_{T, l}\right) \delta\left(\theta_{R}-\theta_{R, l}\right) \\
& \times \delta\left(\varphi_{R}-\varphi_{R, l}\right)
\end{aligned}
$$

where $A_{l}$ defines the gain of the $l$ th path, $\left(\theta_{T}, \varphi_{T, l}\right)$ defines the angle of departure and $\left(\theta_{R}, \varphi_{R, l}\right)$ defines the angle of arrival.

The MIMO channel matrix relates the signals that drive the transmit transducer array, $x(t)$ and the signal from the receive array that is down-converted to produce a baseband signal, $\mathrm{y}(\mathrm{t})$. Consider a MIMO system with $\mathrm{n}_{\mathrm{T}}$ transmit and $n_{R}$ receive antennas. The time-varying channel impulse response between the jth $\left(j=1,2, \ldots, n_{T}\right)$ transmit antenna and the $i$ th $(i=1$, $\left.2, \ldots, n_{R}\right)$ receive antenna is denoted as $h_{i j}(\tau, t)$. This is the response at time $t$ to an impulse applied at time $t-$ $\tau$. The composite MIMO channel response is given by the $\mathrm{n}_{\mathrm{R}} \times \mathrm{n}_{\mathrm{T}}$ matrix $\mathrm{H}(\tau, \mathrm{t})$ as in equation (2)

$$
H(\tau, t)=\left[\begin{array}{cccc}
h_{1,1}(\tau, t) & h_{1,2}(\tau, t) & \ldots & h_{1, n T}(\tau, t) \\
h_{2,1}(\tau, t) & h_{2,2}(\tau, t) & \ldots & h_{2, n T}(\tau, t) \\
\vdots & \vdots & \ddots & \vdots \\
h_{n R, 1}(\tau, t) & h_{n R, 2}(\tau, t) & \cdots & h_{n R, n T}(\tau, t)
\end{array}\right]
$$

The matrix elements or channel gains are complex numbers that correspond to the attenuation and phase shift that the wireless channel introduces to the signal reaching the receiver with delay $\tau$. The effects of the antenna and bandwidth are also included in the channel matrix. The vector:

$\left[h_{1, j}(\tau, t), h_{2, j}(\tau, t) \ldots h_{n R, j}(\tau, t)\right]^{\mathrm{T}}$ is referred to as the spatio-temporal signature induced by the $j^{\text {th }}$ transmit antenna across the receive antenna array. The inputoutput notation of the MIMO system can now be expressed by equation (3)

$\mathrm{y}(\tau)=\mathrm{H}(\tau, \mathrm{t}) \mathrm{x}(\mathrm{t})+\mathrm{n}(\mathrm{t})$

where $\mathrm{x}$ is the $\mathrm{n}_{T} \times 1$ transmitted vector, $\mathrm{y}$ is the $\mathrm{n}_{\mathrm{R}} \times 1$ received vector and $n$ is the $n_{R} \times 1$ additive white circularly symmetric complex Gaussian noise vector. The noise vector is assumed to be independent and identically distributed (i.i.d.) and uncorrelated with the signals. The noise vector could be normalized so that its covariance matrix is the identity matrix.

The analysis of the MIMO channel matrix is a complex mathematical problem. As a result, assumptions are made to simplify solutions of the MIMO channel matrix. Some of these assumptions may be unrealistic for certain environmental conditions [18]. Different antenna configurations may not agree with these assumptions. However, these assumptions aid in the determination of the channel matrix. Assumptions made may be listed as follows:

i. For a narrowband MIMO channel, the elements of $\mathrm{H}$ are complex circularly symmetric Gaussian random variables with zero mean and unit variance $[5,7]$. This is because the fading process is basically stationary and its periods are very much larger than the duration of the fades [5].

ii. The phases of $\mathrm{H}$ may be uniformly distributed over $[0,2 \pi]$.

Iii The elements of $\mathrm{H}$ may be independent. This is true for environments with a high degree of scattering and also substantial angular spread at both transmit and receive sides.

\subsection{Mimo Channel Model Classifications}

MIMO channel models can be classified in terms of the channel type being considered or in terms of the variation in channel characteristics with respect to time. Therefore the MIMO channel could be classified as narrowband channel models for flat-fading channels or wideband channel models for frequencyselective MIMO channels [6]. The narrowband channels are also considered to be quasi-static, static or time invariant since the rate of time variation within the channel is very small compared to any useful signaling rates that could be considered. On the contrary, with a lot of motion within the wideband channels, the wideband channel models are considered to be time varying. Motion may include the movement of objects or scatterers in the channel or movement of the transmitter, receiver or both. 


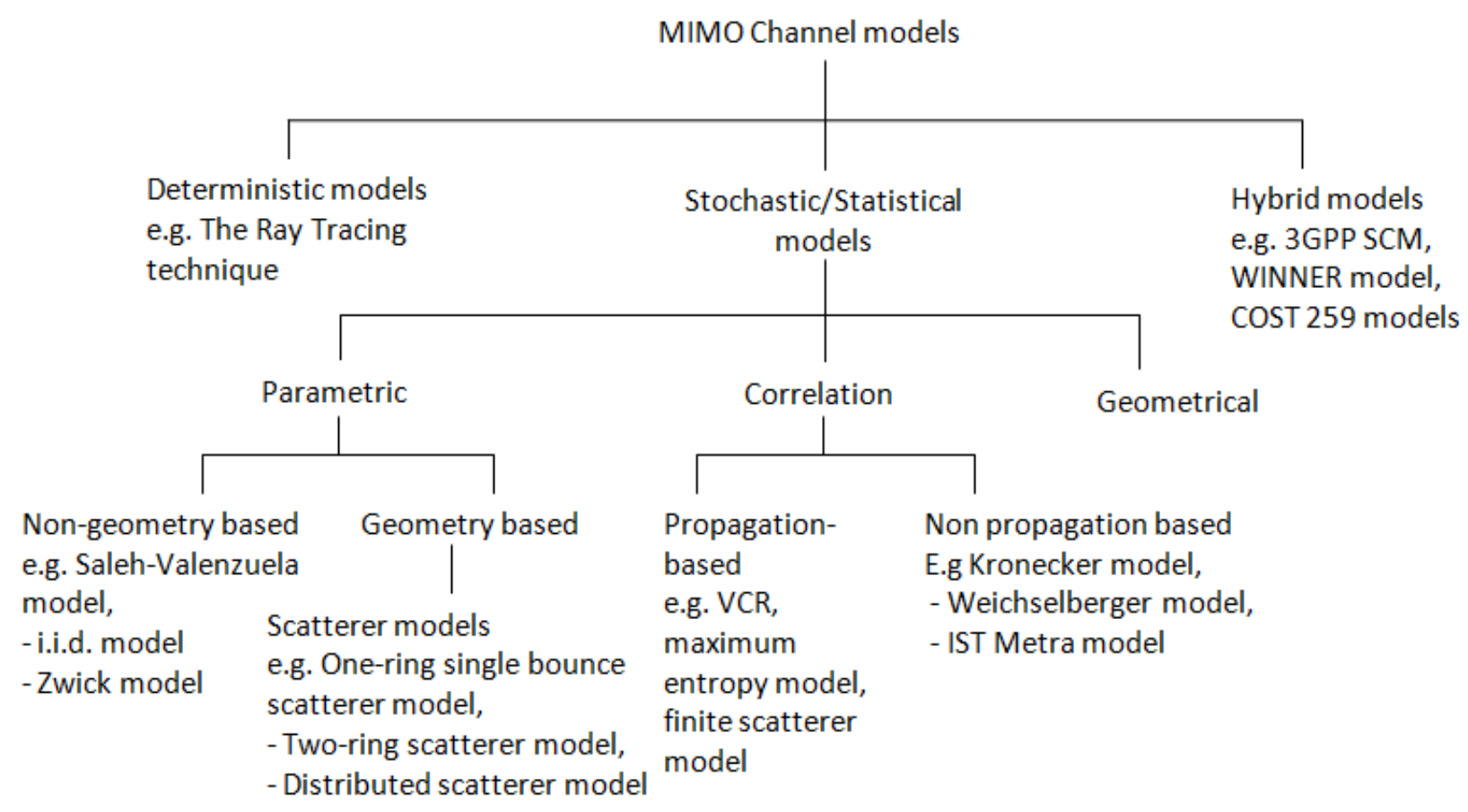

Figure 1: Classification of MIMO channel models

Therefore, the channel impulse response, $h$, is dependent on time. MIMO channel models have also been classified in literature as physical models and analytical models $[3,6]$. A classification of the MIMO channel model is discussed in Figure 1. Various spatial channel models have been developed for the modeling and characterization of the MIMO channel impulse response. A detailed look at some existing MIMO channel models and their limitations are carried out in this study. The overview of spatial channel models for antenna array communications and their drawbacks has been studied extensively in literature. However, most of the limitations studied were done with respect to smart antenna (SIMO and MISO) systems [4].

\section{EXISTING MIMO CHANNEL MODELS}

\subsection{Deterministic/Measurement Based Models}

Field measurements otherwise called deterministic MIMO channel models are models that result from measurement campaigns. These measurements clearly define information regarding the potential performance of a particular propagation environment. The Ray tracing technique which applies Geometric Optics (GO) and the Uniform Theory of Diffraction (UTD) is the most frequently used measurement method. GO is often used to track the propagation of the electromagnetic waves through the propagation environment. GO is an approximate field measurement method for estimating a high frequency electromagnetic field $[1,2,3]$. The ray tracing tool uses information about the environment (geometrical and electrical properties), antennas (position, radiation pattern, polarization and carrier frequency) and electromagnetic interactions to determine the directional complex channel impulse response[2, 6]. Data collected during such measurements are recorded and investigated to properly characterize the MIMO propagation channel. Characterizing the MIMO channel using field measurements is the bedrock to the validation of all stochastic models $[1,6]$. This method is very accurate for particular scenarios compared to other channel model methods. Drawbacks to the deterministic method of MIMO channel modeling include its high cost, difficulty in calibration, time consumption, large computation time, the complexities involved in experimentation and the fact that the equipment needed may not be readily available. In addition, the location of scatterers is usually taken form a specific geographical database $[1,2]$. This implies that measurements resulting from deterministic simulations cannot be generalized since these simulations represent specific propagation scenarios.

\subsection{The I.I.D Model}

The Independent and Identically Distributed (i.i.d) MIMO channel model investigates the use of multielement over the additive Gaussian channel with and without fading for a single user [7].Due to the simplicity of the model, it is widely used in literature. 
This model assumes a random channel matrix with zero mean, independent and identically distributed entries. The channel is assumed to be spatially white, occurring in an area with rich scattering and enough spacing within antenna elements[1, 18].With these characteristics, the channel fade distribution is said to be statistically independent and therefore do not correlate. However in reality, most practical systems exist in an environment with few scatterers and small antenna spacings between transmit and receive sides [18]. This implies that there exists strong correlation between the elements of the channel matrix and mutual coupling between the elements of the antenna array on both link ends. Therefore, the i.i.d model and its capacity gains can be said to exist only in theory and are useful for theoretical evaluations.

\subsection{Geometrically Based Models}

Geometrically based models are physical models [6]. These models apply the fundamental laws of wave propagation as well as the geometry of the propagation environment to define channel parameters in a realistic way [14]. The choice of parameters may include the AoA of the transmitted signal to the receiving end, AoD of the received signal from the transmit side, the Time-of-Arrival (ToA) of the transmitted signal, the Azimuth Spread (AS) to determine the heights of the impinging signals. These models are usually supported by measurement or stochastically chosen scatterer locations so as to suit varying propagation conditions [6]. Scatterer distributions completely describe the distribution of both effective and distant scatterers or objects that affect the propagation channel. Therefore the model could be used to completely characterize the MIMO channel. The stochastic distribution of scatterers (diffuse or discrete) aims to mimic the real life distribution of scatterers. Scatterers could therefore be placed in a deterministic way [4] or at random [1, 8]. Geometrical-based stochastic/scatterer models also determine the distribution of scatterers and scatterer density within the propagation environment [28].One drawback of the geometrical representation of the MIMO channel is that it uses only the antenna properties in constructing the channel. Examples arethe two-ring scatterer model, the one-ring model with single bounce scattering [6], the elliptical model and the cylindrical models.

The one-ring model is a geometrically based stochastic model for the macro-cellular wireless network $[1,2$, 9]. The transmitter is assumed to be elevated above all surrounding scatterers while the receiver is surrounded by effective or local scatterers. Therefore the one ring model assumes that signals reach the receiver in different directions according to predetermined statistical distributions. Different scatterer distributions around the receiver have been proposed and this depends on the type of environment being studied [8, 9]. An efficient scatterer distribution is required to determine the AoA and AoD since their value depends on the environment surrounding the transmitter and receiver. The delay of a multipath component that propagates through local scatterers can be determined if the scatterer locations or the distance of the scatterers are known. An illustration of the onering model is shown in Figure 2.

It is assumed that there is no line of sight path between the transmitter and the receiver. The above geometry illustrates a channel with two transmit and two receive antennas. The antenna spacing between transmit and receive sides is defined by $\delta_{\mathrm{Tx}}$ and $\delta_{\mathrm{Rx}}$ respectively. Other dimensions of the model are as defined in $[1,9]$. This model assumes only single scattering with all scatterers having equal power. The correlation and correlation matrices between pairs of antenna elements can be determined from the model. The level of correlation determines the performance of spatial diversity methods. Although the model predicts correlations between any two elements of the antenna array, it is inadequate for a complete description of all observed channel effects on the MIMO channel. This representation is inconvenient for the realization of a closed-form analysis of the spacetime code behaviour of the MIMO channel.

The two-ring model illustrates two rings that are drawn with centers a ttransmit and receive ends of the link as shown in figure3.

Their radii represent the average distance between each communication node and their respective scatterers $[1,19,20] . S c a t t e r e r s$ are then placed randomly on these rings. The two-ring model is based on the assumption that scatterers surrounding the transmitter and receiver control the AOD and AOA respectively. The two-ring single bounce scattering model is easily tractable based on how scatterers are distributed and clustered [1]. The Elliptical MIMO channel model defines the distribution of effective scatterers on the eccentricity of an ellipse. The model is suitable for channel types in which the antenna heights for both transmitter and receiver are at the same height. 


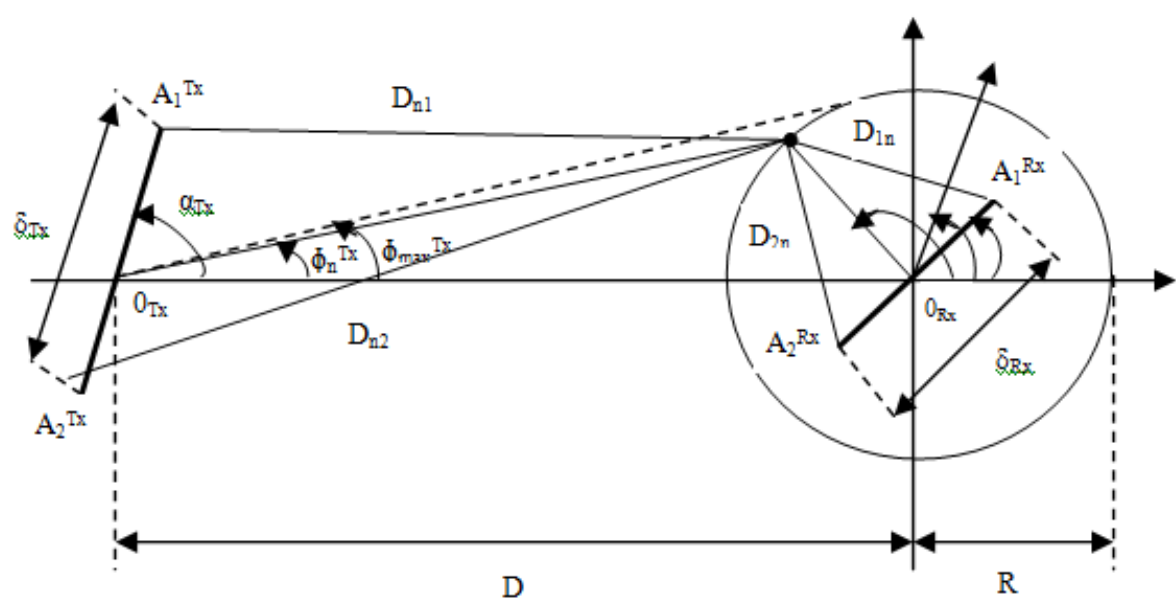

Figure 2: The one-ring model with elevated transmitter and scattering around the receiver [9]

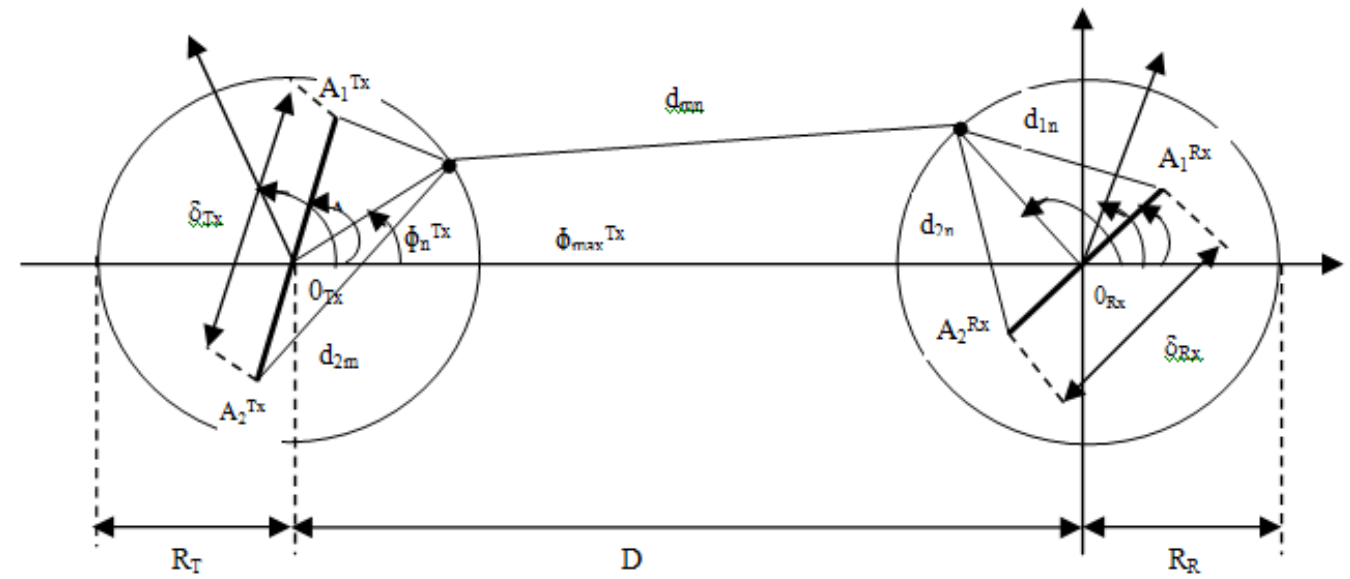

Figure 3: The two-ring model showing the transmitter and receiver at the same or almost the same elevated position.

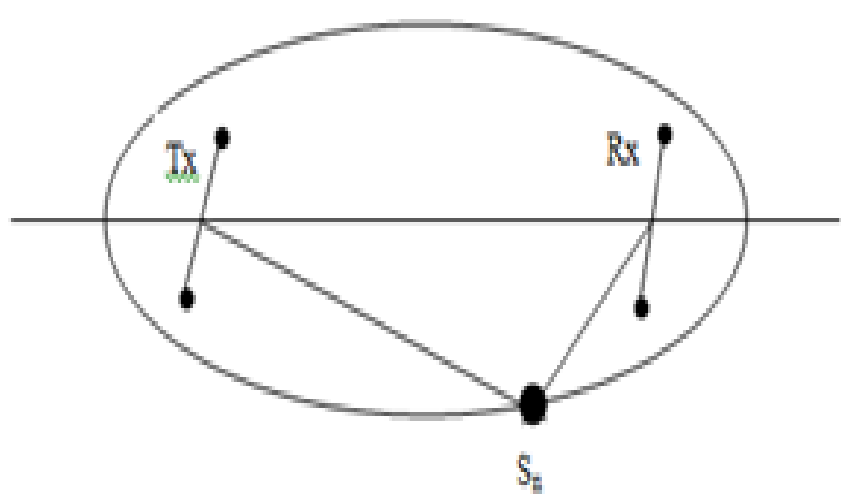

Figure 4: the Elliptical Mimo channel model

This model is also suitable for channels with a dominant line-of-sight component, reflective surfaces and mobile-to-mobile communication systems $[8,10$, 14]. A combination of the elliptical model with the two-ring model has been used to illustrate both mobile and stationary scatterers [14].

An illustration of the elliptical MIMO channel model for a $2 \times 2$ channel is shown in figure $4 . \mathrm{Tx}, \mathrm{Rx}$ and $\mathrm{S}$ represent the transmitter, receiver and effective scatterers. The number of effective scatterers is given as $n$, where $n=\{1,2,3, \ldots\}$.

The geometrical models discussed in section 2.3exhibit single bounce scattering. Single scattering has a number of limitations which result from some assumptions made prior to modeling. Transmit and receive scatterers (eigenmodes) paired arbitrarily take part in the propagation of only one wave. Therefore the model may be assumed to create a channel that behaves like a min $(n, m)$ properly separated and parallel SISO channels. Such a channel should exist in an environment with rich scattering. This implies that single scatterers are the cause for diagonality in the channel covariance matrix [17].

In contrast to the above assumption, in reality, signals reflected by the scatterers at the receive side are not independent. This implies that the channel coefficient cannot be zero-mean complex Gaussian, a characteristic of channels with rich scattering and a diagonally dominated structure $[8,17]$. Consequently, the channel covariance matrix cannot completely describe the MIMO channel. 
In addition, scatterers within the propagation environment are assumed to be re-radiating in all directions and are modeled based on some assumed random complex scattering co-efficient [12, 13]. However, it is difficult to assign realistic scattering coefficients [13]. Therefore the single bounce model cannot adequately model all observed channel effects. To overcome the aforementioned problems, various geometrical-based stochastic models (GBSM) which include both single- and double-bounced rays with different energies have been proposed in literature [14]. The model can be adapted to any type of environment - picocell, microcell, macrocell environments. To achieve this adaptation, the shape of the scattering disc could be changed accordingly.

A proposition for the geometrical scatterer model was given in [15].Combined beamforming and the diversity model were used to determine the lower bound on the error correlation matrix. The beamforming model is a geometrical based scattering model that employs the angular resolvability of a uniform linear antenna array and the angular domain representation of signals. The diversity model focused on the multipath environment, amplitude variations and the signal power. A combination of beamforming and diversity ensures that signals from multiple transmit antennas are properly selected by multiple receive antennas. Obtaining a solution for the spatial channel response using this combined approach may lead to complex intractable expressions and so many assumptions.

In [4], a geometrical scatterer model based on the environment propagation conditions and defined by a spatial scatterer density function was proposed for the smart antenna system. In $[4,15]$, the AOA and AOD are considered to be slowly varying features of the channel. This claim is justified by the fact that even in mobility; the variations of the AOA and AOD are smaller than the angular resolution of the arrays $[10$, 11].

Since these geometric models are based on the concept of scattering within the propagation environment, a complete idea of the location of the spatial scatterer density function is required to provide an accurate model of the channel. This implies that the GBSM is less accurate compared to the deterministic models. The model also needs experimental data for proper validation. Nevertheless, the GBSM remains the best model that can easily be adapted to all environmental configurations since by changing the shape of the scattering disc and the scatterer densities one can mimic real life scenarios $[3,14]$. The GBSM is cost effective. The only requirement is to model the system according to the laws of wave propagation. The GBSM has a lower computation time and lesser complexity since only effective scatterers are required in simulation.

\subsection{Propagation Based Models}

The Virtual Channel Representation (VCR) model employs a virtual partitioning of the spatial environment to characterize the MIMO channel as shown in figure 5 .

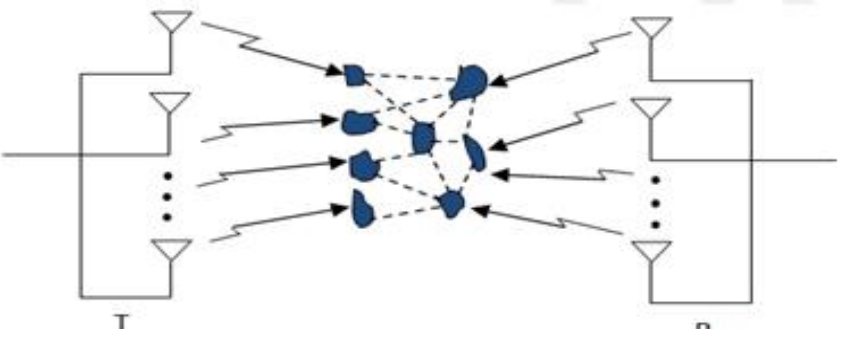

square Figure 5: The Viritual Channel Model [13]

An equivalent virtual representation of the MIMO channel is obtained through the application of a spatial discrete fourier transform (DFT). The virtual channel matrix could be considered as an image of the physical scattering environment with elements that are assumed to be approximately independent. The VCR model can be expressed as $[13,17]$

$$
H_{\text {virtual }}=A_{r}\left(\widetilde{\Omega}_{\text {virtual }} \odot \quad G\right) A_{t}^{T}
$$

where $A_{r}$ and $A_{t}$ are steering matrices that are channel independent DFT matrices of size $n \times n$ and $\mathrm{m} \times \mathrm{m}$ respectively. $\widetilde{\Omega}$ is the element-wise root of the power coupling matrix $\Omega$ with positive and real-valued entries that specify the mean amount of energy that is matched between an mth transmit and $n t h$ receive direction. The coupling matrix affects the channel capacity as well as the degree of diversity in the channel[18]. $G$ is an i.i.d. zero-mean complex Gaussian matrix. The structure of the coupling matrix reflects the spatial arrangement of scatterers. The VCR model completely models the joint angular power spectrum of the MIMO channel which depends on the actual antenna configuration and the number of antennas [19]. Therefore the angular resolution and accuracy of the VCR model depends on the actual antenna configuration of the MIMO system. The accuracy of the model increases with increase in the number of antennas. The VCR model is restricted to Uniform Linear Arrays (ULAs). This means that VCR can only be applied to specific systems. In addition, a virtual partitioning of the spatial domain that is fixed and 
pre-defined is used to characterize the MIMO channel. This fixed and pre-defined spatial direction implies that the model tends to characterize the MIMO channel with more multipath components than the channel actually has, thereby reducing channel correlation and increasing the channel capacity [13, 19]. Thus, the channel capacity is often overestimated. The VCR model has been applied to determine the capacity of MIMO channels with uniform linear antenna arrays at both transmitter and receiver side for frequency-selective channels[13].The analysis was based on the assumption that there may be limited scattering in a given propagation environment and limited spacing between antennas. This assumption is realistically correct since it is obvious that rich scattering is a very simplifying assumption found in very few environments. The proposed model uses a virtual representation that directly relates the channel to the physical scattering environment with a channel matrix that is presented as a continuum of paths [13]. The need for a virtual representation of the channel was not clearly expressed. From the authors, perspective, a key property to be exploited in the channel model is that the elements of the virtual channel matrix are assumed to have independent entries. This assumption was also made in earlier correlation-based models like the Kronecker channel model. The Kronecker model is an i.i.d. model that assumes that scattering at transmitter and receiver is independent as well as rich scattering. Therefore there is no clear distinction between the assumptions made regarding an i.i.d. channel and the virtual channel matrix. Other propagation based models are the maximum entropy model [29] and the finite scatter model [9].

\subsection{CORRELATION BASED MODELS}

The correlation matrix model is the simplest strategy for modeling the MIMO channel [1].The correlation model employs the effect of antennas, the physical channel and matched filtering. The model depends on the assumptions made about the specific antenna types and array configurations. Early MIMO channel models assumed an independent and identically distributed (i.i.d.) multivariate complex normal (MCN) distribution where the covariance matrix is an identity matrix $[1,2,7]$. In MIMO channel modeling the MCN distribution may be used because of its simplicity and compatibility with Rayleigh fading [7]. The correlation model provides a fairly general result for the form of the covariance matrix. It also gives an insight into the correlation between transmit and receive antennas (spatial diversity) and antenna spacing. However, the flexibility of the model is limited. Examples of correlation models include the Kronecker model, the Weischelberger model and the METRA model. These models are based on the spatial (antenna) or power correlation between the transmit array and the receive array of the same communication link. The limitations of each of these models will be discussed in detail.

The Kronecker model was developed mainly for i.i.d Rayleigh distributed channels. The model represents correlations that arise with spatial diversity due to antenna proximity. The model is represented as $[13$, 19].

$$
H=R_{R}^{1 / 2} H_{\text {i.i.d. }} R_{T}^{1 / 2}
$$

where $R_{T}$ and $R_{R}$ are transmit and receive correlation matrices respectively. $H_{\text {i.i.d. }}$ is an $\mathrm{n}_{\mathrm{R}} \times \mathrm{n}_{\mathrm{T}}$ matrix of i.i.d. complex normal elements. This model is analytically friendly but not universal. The validity of the Kronecker model lies on the fact that the transmit correlation coefficients are independent of the receive antennas. In other words, the model describes the spatial correlation of both ends of the link as separable [19]. It overlooks the interdependence of both transmit and the receive side of the MIMO channel in communication - one of its greatest drawbacks. The correlation matrices of the Kronecker model are obtained with the assumption that the signal covariance of the other end of the link is spatially white. This implies that the transmitter does not affect the spatial properties of the received signal at all and there are no diagonally dominated coupling matrices. The more diagonal the structure of the coupling matrix becomes the higher its channel capacity estimates. This limitation of the Kronecker model leads to wrong capacity estimates and a difference between the model and measured data. The Kronecker model lacks the essential degrees of freedom that are found in more general MIMO channel models. This is due to a mismatch between its coupling matrix and its entries. Additional information is therefore required for the application of the Kronecker model to typical environments.

The Weichselberger model merges the advantages of both the Kronecker model and the Virtual Channel Representation (VCR) model. The Weichselberger model can be defined as an extension of the VCR model that applies arbitrary antenna geometries without the seperability of the Kronecker model. The Weichselberger can be expressed as $[17,18,19]$ 


$$
H_{\text {weichsel }}=U_{r}\left(\widetilde{\Omega}_{\text {weichsel }} \odot \quad G\right) U_{t}^{T}
$$

where $\boldsymbol{U}_{\boldsymbol{r}}$ and $\boldsymbol{U}_{\boldsymbol{t}}$ define the spatial eigenbasis at the transmit and receive side respectively. $\widetilde{\Omega}$ is the element-wise square root of the power coupling matrix $\Omega$ and $\mathrm{G}$ is an i.i.d. zero-mean complex Gaussian matrix. The Weichselberger model ensures that there exists a joint correlation between the transmit antenna array and the receive antenna array. The model bases its argument on the assumption that there should be a connection and mutual interdependence between the spatial correlations of both ends of the link. The introduction of a coupling matrix that presents a diagonal structure is one step to the inclusion of joint spatial correlation in the model. This diagonal structure in the coupling matrix ensures transmit and receive diversity and also supports spatial multiplexing $[13,18]$. In certain realworld scenarios, the Weichselberger model may be accepted as general or realistic. However, the model fails to characterize some propagation properties of the MIMO multipath channel. The Weichselberger model does not account for the joint AoA)/AoD angular power spectrum of impinging signals. The model does not also account for mobility within the channel or between link ends.

The European Union IST METRA (Multi Element Transmit Receive Antenna) model was proposed for frequency-selective fading channels $[20,21]$. The METRA model is an analytical model whose main strength lies in the spatial power correlation between the antenna arrays at the receiver and at the transmitter. The power correlations at the receiver and at the transmitter are modeled independently. This implies that transmit and receive side of the link are spatially uncorrelated. The joint spatial power correlation of the MIMO channel can be determined by taking the kronecker product of the correlation matrices at both link ends. The METRA model is also characterized by the Power Delay Spectrum (PDS) which evaluates the channel impulse response for frequency-selective channels, the Doppler Spectrum which specifies the time domain characteristics of the model and the Power Azimuth Spectrum (PAS) from which the correlation matrices and the Azimuth Spread can be determined. Since the METRA model depends on the power correlation matrix, details about the carrier phase is unaccounted for. This is because the power correlation coefficients do not take the phase information into account [12]. As a result, there is great difficulty in matching capacity. Also the polarization of the antenna arrays was not considered.
In addition, there is no clear coupling between transmit and receive correlation matrices in the model.

\subsection{NON GEOMETRICAL BASED MODELS}

Non-geometrical based models are also referred to as statistical models. Statistical models determine the parameters of a channel without applying any underlying geometry. Statistical models have been applied in literature for the analysis of both indoor and wideband propagation scenarios $[11,12,16,20$, $21,22]$. Statistical models are analytical models that classify the propagation environment based on a combination of experimental validations, analytical representations (probability density functions to mimic the behaviour of real world scenarios) and electromagnetic wave propagation theories. For these models, multipath propagation is usually modeled as a two-way directional propagation between the transmitter and the receiver[23]. The statistical models have the advantages of flexibility, simplicity in analysis, ability to reflect the time variant nature of the MIMO channel and extension to a large number of propagation scenarios[11, 12]. A disadvantage to the use of statistical models is that there is a leap in the complexity of the channel model when extended to include more parameters. Therefore closed-form solutions are often difficult to obtain without making ambiguous assumptions. To overcome this limitation, channel measurements or simulations are often needed to determine pertinent parameter values. In some literatures, statistical models are often represented as a hybrid of both the deterministic and analytical modeling approaches. The SV model is a case of statistical model that was validated experimentally. Although, the SV model was developed for smart antennas, it has been extended in literature to accommodate MIMO channels [12]. The received signal rays are modeled as arriving in clusters. Both rays and clusters arrival times are modeled as a Poisson arrival process with different but fixed rates. The rays have independent uniform phases and Rayleigh distributed amplitudes. Extension to the SV model is referred to as the SV model with angle or SVA model. The SVA model characterizes the channel by representing each MPC in terms of its amplitude, arrival time, AoA and AoD. Another advantage of statistical models is the fact that they are accurate and adequate in developing, evaluating and optimizing adaptive directional antenna concepts [22]. These directional antenna 
concepts are limited to the path directions of the MIMO multipath components. The mobility of nodes in the network is unaccounted for. Consequently, statistical models do not consider mobile nodes.

\subsection{Hybrid/Standardized Mimo Channel Models 2.7.1 Cost 259}

The European Cooperation in the field of Scientific and Technical Research (EURO-COST) COST 259 model is a directional, geometrically based stochastic MIMO channel model. The COST 259 MIMO model focuses on the mobile and its attendant energy emissions before impinging on the base station. Thus the model applies the property of beamforming to different environmental conditions [30]. The COST 259 model is defined for different environments which may have several propagation scenarios and different parameter sets[30, 32]. Environments considered include the pico-, micro-, and macro-cellular environments. Channel modeling for each environment follows a different approach. Measurements are taken with respect to the mobile terminal for both LOS and NLOS cases since measurements confirm that energy comes from the direction of the mobile. Parameters considered in the model are the angular parameters (angular spread, Azimuth power distribution, Direction of Arrival) and selectivity parameters (excess delay spread, delay window) as well as the wideband channel impulse responses. Measurements in the COST 259 model assume that the channel is stationary. This is a drawback to the use of the model for frequency selective channels.

\subsubsection{GPP SCM}

The 3rd Generation Partnership Project Spatial Channel Model is a ray-based, geometrically-based stochastic MIMO channel model with a centre frequency of $2 \mathrm{GHz}$ and a system bandwidth of $5 \mathrm{MHz}$. This model was formed by the combined efforts of two adhoc groups - the 3GPP and the 3GPP2 with the aim of developing a standardized model for array communications $[2,6]$. A key parameter in the model is the number of subpaths (rays), their spatial amplitude and temporal variations. Various subpaths form a path. Each path is formed by summing together a number of subpaths. A collection of 6 paths and 20 subpaths were used to determine the channel model. All paths are assumed independent and each is characterized by its own spatial channel parameters. The channel matrix is determined following three basic steps: environment specification, simulation parameter identification and the generation of channel coefficients. The "drop" concept is applied in the model for fast fading links with respect to the speed of the mobile. The drop is defined as one simulation run over a short period of time $[2,6]$. To justify this claim, it was noted that the components of the channel coefficients are not necessarily resolvable in the time domain since the time difference between successive paths may be less than a chip period [2].The channel model allows any type of antenna configuration. To allow comparison of different antenna scenarios, the transmit power of a single antenna case is the same as the total transmit power of a multiple antenna case $[2,30]$.A drawback to the 3GPP SCM model is that it involves the determination of a large volume of simulations and parameters in the model. This may increase computational load. In addition the channel modeling is so generalized and this may give inaccurate data in some fields.

\subsubsection{IST Winner}

The IST WINNER MIMO channel models combine the characteristics of existing MIMO channel models (e.g. 3GPP, COST 259 and METRA) in order to solve open issues found in these models[32]. The WINNER model is divided into link-level and system-level evaluations. The link-level evaluations are basically for calibration between antenna arrays while the system-level evaluations are for the solution to problems associated with channel transmission and reception. The WINNER models cover various environmental scenarios such as the indoor, suburban, urban and rural environments.

\section{MIMO MOBILITY MODELS}

MIMO mobility models are usually developed from a reference model[14, 23, 24, 25, 26]. The reference model could be a geometrical model, stochastic, SISO channel model or a combination of various models. From a reference point, the effects of Doppler, angular spread and the time-varying nature of the complex environment can be modeled. Mobile channels could include fixed-to-mobile nodes (e.g. a fixed base station and mobile station that both have multiple antenna systems) or mobile-to-mobile nodes (e.g. an intelligent ad-hoc network in which both the transmitter and the receiver are in motion). Due to the movement of both transmitter and receiver in a mobile to mobile communication system, the received signal experiences Doppler shifts. Therefore, the effect of Doppler spread on the channel must be considered 
for mobile MIMO fading channels in addition to other channel effects. The movements of nodes in the channel also increase the time varying nature of the fading envelope. Some of the modeling methods employed in the modeling of mobile MIMO channels include; the geometrical method (with regular or irregular geometries), stochastic modeling method and the deterministic modeling method. The irregular shaped geometrical-based models were used in [23]. The aim of this model was to represent the time varying nature of the mobile MIMO channel in a more realistic manner. The model was parameterized using experimental results carried out in both highways and rural areas. Regular shaped geometries include the one-ring, two-ring, two-cylinder, the elliptical and the two-ring elliptical model[14, 24, 25, 26]. These regular geometries depict the shape of the scattering region around transmit and receive antennas. It has been argued in [23] that the regular geometries do not depict the non-stationary nature of the scattering region. However the two-ring elliptical model developed in [14] was used to mimic both the stationary and non-stationary nature of scatterers around the antennas. These regular geometrical models are useful for analytical studies and the simulation of space-time cross correlation functions. Stochastic channel model that has been used in literature is the tapped-delay line model[27]. This model assumes a fixed Doppler spectrum for every delay in a bid to represent the non-stationary nature of the mobile MIMO channel.

\section{OPEN ISSUES ON MIMO CHANNEL MODELLING}

For over 15 years, so much theoretical works concerning channel modeling in multiple antenna systems has been done. Notwithstanding, the real-life implementation of such channel models are still in their infancy. This is as a result of the many ambiguous assumptions that have been made in channel modeling. Assumptions have been made on the effect of scatterers, channel capacity and model standardization. One of the greatest challenges in the modeling of the MIMO channel is the effect of scatterers within the channel. It is very important to classify completely the effects of scatterers and scatterer densities within the channel. Most of the works done in literature for both LOS and NLOS channels consider only local scatterers as effective scatterers. This is a very simplifying assumption. For instance, in the NLOS case, the transmit signal travels wirelessly over various distant and local scatterers.
These scatterers could be densely or diffusely distributed. Proper classification and study of the effect and type of scatterering is very important in MIMO channel modeling. Secondly, the theoretical capacity of the MIMO system is often overrated. For areas without rich scatterering, there should be a realistic channel capacity measure devoid of ambiguous assumption. Thirdly, a standard reference model which is generally accepted by all standardizing bodies is needed for the validation of new MIMO channel models. This model should be computationally tractable, generic, and easy to understand.

\section{CONCLUSION}

For several years, channel modeling for MIMO wireless system has been a great challenge. Various channel characterizations and model classifications have been proposed in literature. Channel models can be broadly classified into the deterministic models (models determined through experimentation), stochastic models (models determined through analytical or statistical representations) and hybrid models (models that combine both the deterministic and stochastic methods). All existing models have their accomplishments and issues. Thus, for each model discussed, these issues and accomplishments have been detailed for ease of understanding. Finally, open issues on MIMO channel modeling were enumerated.

\section{REFERENCES}

[1] Jensen, M. A.and Wallace, J. W., Space-Time Processing for MIMO Communications, John Wiley and Sons, 2005.

[2] Tsoulos, G., MIMO System Technology for Wireless Communications, Taylor and Francis,, London, 2006.

[3] Carlos, P.,Yannis, P., Rodolphe, V, and Pierre, C. "Sensitivity of the MIMO Channel Characterization to the Modelling of the Environment" IEEE Transactions on Antennas and Propagation, Vol. 57, Issue 4, April 2009, pp 1218-1227.

[4] Ertel, R. B, Cardieri, P. et al "Overview of Spatial Channel Models for Antenna Array Communication Systems" IEEE Personal Communications, Vol. 5, Issue 1, February 1998, pp 10-22.

[5] Goldsmith, A Ali Jafar, S. et al "Capacity Limits of MIMO Channels" IEEE Journal on Selected Areas In Communications, Vol. 21, Issue 5, June 2003, pp 684702 .

[6] Almers, P., Bonek, E., Burr, A., Czink, N., Debbah, M., Degli-Esposti, V., Hostetter, H., Kyosti, P., Laurenson, D., Matz, G., Molisch, A. F., Oestges C. and Ozcelik, H. "Survey of Channel and Radio Propagation Models for Wireless MIMO Systems" EURASIP Journal on Wireless 
Communications and Networking, Vol. 2007, February 2007.

[7] Telatar, E. "Capacity of Multi-antenna Gaussian Channels", AT\&T-Bell Labs Internal Tech. Memo., June 1995.

[8] Molisch, A. F. "A Generic Model for MIMO Wireless Propagation Channels in Macro- and Microcells" IEEE Transactions on Signal Processing, Vol. 52, Issue 1, January 2004, pp 61-71.

[9] Pätzold, M. and Hogstad, B. 0. "A Space-Time Channel Simulator for MIMO Channels Based on the Geometrical One-Ring Scattering Model", IEEE 60th Vehicular Technology Conference, 26-29 September, 2004, pp 144-149.

[10] Liberti, J. C. and Rappaport, T. S. "A Geometrically Based Model for Line-of Sight Multipath Radio Channels" IEEE 46th Vehicular Technology Conference, 28 Apr - 1 May, 1996, pp 844-848.

[11] Saleh, A. and Valenzuela, R. "A Statistical Model for Indoor Multipath Propagation" IEEE Journal on Selected Areas in Communication, Vol.5, Issue 2, February 1987, pp 128-131.

[12] Jensen, M. A. and Wallace, J. W. "Statistical Characteristics of Measured MIMO Wireless Channel Data and Comparison to Conventional Models" IEEE 54th Vehicular Technology Conference, 7-11 October, 2001, pp 1078-1082.

[13] Veeravalli, V., Liang, Y., and Sayeed, A. M. "Correlated MIMO Wireless Channels: Capacity, Optimal Signalling and Asymptotics" IEEE Transactions on Information Theory, Vol. 51, Issue 6, June 2005, pp 2058-2072.

[14] Cheng, X., Cheng-Xiang Wang, Laurenson, D. I., Salous, S., and Vasilakos, A. V. "An Adaptive Geometry-Based Stochastic Model for Non-Isotropic Mobile-to-Mobile Channels" IEEE Transactions on Wireless Communications, Vol. 8, Issue 9, September 2009, pp 4824-4835.

[15] Simeone, O. and Spagnolini, U. "Lower Bound on Training -Based Channel Estimation for FrequencySelective Block-Fading Rayleigh MIMO Channels", IEEE Transactions on Signal Processing, Vol. 52, Issue 11, November 2004, pp 3265-3277.

[16] Zwick, T., Fischer, C. and Wiesbeck, W. "A Stochastic Multipath Channel Model Including Path Directions for Indoor Environments" IEEE Journal on Selected Areas in Communication, Vol. 20, Issue 6, August 2002, pp 1178-1192.

[17] Weichselberger, W., Herdin, M., Ozcelik, H. and Bonek, E. "A Stochastic MIMO Channel Model with Joint Correlation of Both Link Ends" IEEE Transactions on Wireless Communications, Vol. 5, Issue 1, January 2006, pp 90-100.

[18] Gesbert, D., Bolcskei, H., Gore, D. A. and Paulraj, A. J. "Outdoor MIMO Wireless Channels: Models and Performance Prediction" IEEE Transactions on Communications, Vol. 50, Issue 12, December 2002, pp 1926-1934.

[19] Ozcelik, H., Czink, N. and Bonek, E."What Makes a Good MIMO Channel Model?" IEEE 61st Vehicular Technology Conference, 30 May-1 June, 2006, pp 156160.
[20] Kermoal, J. P., Schumacher, L. and Pedersen, K.I. “A Stochastic MIMO Radio Channel Model With Experimental Validation" IEEE Journal on Selected Areas in Communications, Vol. 20, Issue 6, August 2002, pp 1211-1226.

[21] Pedersen, K., Andersen, J. B., Kermoal, J. P. and Mogensen, P. "A Stochastic MIMO Radio Channel Model for Evaluation of Space-Time Coding Algorithms" IEEE 52nd Vehicular Technology Conference, 24-28 September, 2000, pp 893-897.

[22] Klein, A. and Mohr, W. "A Statistical Wideband Mobile Radio Channel Model Including the Directions-ofArrival' SIEMENS AG, Munich Germany, 1996.

[23] Karedal, J., Tufvesson, F., Czink, N., Paier, A., Dumard, C., Zemen, T., Mecklenbrauker, C. and Molisch, A. "A Geometry-Based Stochastic MIMO Model for Vehicleto-Vehicle Communications" IEEE Transactions on Wireless Communications, Vol. 8, Issue 7, July 2009, pp 3646-3657.

[24] Pätzold, M., Hogstad, B. O., Youssef, N. and Kim, D. "A MIMO Mobile-to-Mobile Channel Model:Part I - The Reference Model" IEEE 16th International Symposium on Personal, Indoor and Mobile Radio Communications, 2005, pp 573-578

[25] Hogstad, B. 0., Pätzold, M., Youssef, N. and Kim, D. "A MIMO Mobile-to Mobile channel model: Part II - The Simulation Model." in Proc. 16th IEEE Int. Symposium on Personal. Indoor and Mobile Radio Comm., PIMRC 2005, Berlin, 11-14 September, 2005, pp 562-567.

[26] Abdi, A. and Kaveh, M. "A Space-Time Correlation Model for Multi-element Antenna Systems in Mobile Fading Channels" IEEE Journal on Selected Areas in Communications, Vol. 20, Issue 3, April 2002, pp 550560.

[27] Acosta-Marum, G. and Ingram, M. A. "A BER-based partitioned model for a $2.4 \mathrm{GHz}$ vehicle-to-vehicle expressway channel," Wireless Personal Communication, Vol. 37, Issue 3, May 2006, pp 421443.

[28] Oestges, C., Erceg, V., and Paulraj, A. J. "A Physical Scattering Model for MIMO Macocellular Broadband Wireless Channels" IEEE Journal on Selected Areas in Communications, Vol. 21, Issue5, June 2003, pp 721729.

[29] Debbahand, M. and Müller, R. R. "MIMO Channel Modeling and the Principle of Maximum Entropy" IEEE Transactions on Information Theory, Vol. 51, Issue 5, May 2005, pp 1667-1690.

[30] Pajusco, P. "Experimental Characterization of D.O.A at the Base Station in Rural and Urban Area" IEEE 48th Vehicular Technology Conference, 18-21 May, 1998, pp 993-997.

[31] Baum, D. S., Hansen, J. and Salo, J. “An Interim Channel Model for Beyond-3G Systems - Extending the 3GPP Spatial Channel Model (SCM)" IEEE 61st Vehicular Technology Conference, 30 May-1 June, 2005, pp 3132-3136.

[32] Meinilä, J., Jämsä, T., Kyösti, P., Laselva, D., El-Sallabi, H., Salo, J., Del Galdo, G. and Milojevic, M. " $A$ set of channel and propagation models for early link and system level simulations" IST-2003-507581 WINNER D5.1 v1.0. 